\title{
The surgical pathology of pulmonary infarcts: diagnostic confusion with granulomatous disease, vasculitis, and neoplasia
}

\author{
Samuel A Yousem \\ Department of Pathology, University of Pittsburgh Medical Center, Pittsburgh, PA, USA
}

\begin{abstract}
Twenty-three cases of surgically resected pulmonary infarcts sent in consultation were reviewed to evaluate their morphology and to assess reasons for consultation. The morphology of these infarcts demonstrated that only a minority had the classical triangular shape at low magnification $(26 \%)$ whereas the majority were either spherical $(17 \%)$ or had a geographic pattern of necrosis $(35 \%)$. The margin of the infarcted tissue often had a pseudogranulomatous appearance due to palisaded histiocytes, foam cells, or perpendicularly oriented proliferations of fibroblasts and myofibroblasts $(\mathbf{7 4} \%)$ and occasional cholesterol- and hemosiderin-laden giant cells. Basophilic granular karyorrhectic necrosis was seen focally (52\%) as was vascular inflammation (56\%) raising the differential diagnosis of Wegener's granulomatosis or infectious granulomas. These nonclassical features combined with a low incidence of clinical hemoptysis, chest pain and pleurisy, and a primary radiographic diagnosis of 'nodule r/o malignancy' highlight the need to consider thromboembolic pulmonary infarcts in the differential diagnosis of necrotic lung nodules with a histiocytic and fibroproliferative rim. Modern Pathology (2009) 22, 679-685; doi:10.1038/modpathol.2009.20; published online 13 March 2009
\end{abstract}

Keywords: infarct; thromboemboli; lung

Discussions of pulmonary thromboemboli and pulmonary infarcts most often occur in the autopsy suite rather than the surgical pathology laboratory. In fact, some renowned textbooks on pulmonary surgical pathology have failed to discuss the pathology of thromboemboli or infarcts at all, leaving such descriptions to basic pathology textbooks such as Robbins. ${ }^{1}$ We have recently encountered a number of pulmonary infarcts that have been resected surgically, often to exclude neoplasia, which have proven diagnostically problematic to surgical pathologists who have referred them as granulomatous disease, vasculitis, and neoplasia. This precipitated a review of our files and a histologic study of surgically resected thromboembolic pulmonary infarcts to highlight their morphologic spectrum including variations from classical descriptions.

\section{Materials and methods}

Seventy-eight cases of pulmonary infarcts were retrieved from the consultation files and the surgical

Correspondence: Dr SA Yousem, MD, Department of Pathology, University of Pittsburgh Medical Center, Presbyterian Campus, Room A610, 200 Lothrop Street, Pittsburgh, PA 15213-2582, USA. E-mail: yousemsa@upmc.edu

Received 21 October 2008; revised 17 December 2008; accepted 16 January 2009; published online 13 March 2009 pathology archives of the University of Pittsburgh Medical Center between 1994 and 2008. Of these 23 cases were specifically selected as being a result of thromboembolic disease rather than other causes of pulmonary infarction, including neoplasia, infection, vasculitis, amyloid, intravascular foreign bodies, septic infarcts, diffuse alveolar damage, torsion, and amniotic fluid emboli; 10 were reviewed within the last 2 years. Only wedge/ segmental biopsies of the lung were reviewed; four core biopsies of thromboembolic pulmonary infarcts were excluded from consideration.

From 6 to 34 (mean 6) hematoxylin and eosin stain slides were available for review from each case. Ziehl-Nielsen and Gomori methenamine silver stains were reviewed and interpreted as negative in all cases. Verhoeff-van Gieson elastic tissue stains were carried out in 18 cases. Relevant clinical and radiologic features were obtained from medical records and referring pathologists and/or physicians. The University of Pittsburgh Institutional Review Board for protection of human subjects approved the study.

\section{Results}

The clinical features of the 23 patients who comprised this study are summarized in Table 1. 
Table 1 Clinical features of 23 patients with thromboembolic pulmonary infarcts

\begin{tabular}{lc}
\hline Mean age & 54.2 years (range $35-78 ;$ median 48$)$ \\
M/F ratio & $12 / 11$ \\
& \\
Presenting complaints $(N=20)$ & $6(30 \%)$ \\
Cough & $6(30 \%)$ \\
SOB & $4(20 \%)$ \\
Pneumonia & $3(15 \%)$ \\
Hemoptysis & $4(20 \%)$ \\
Chest pain & $4(20 \%)$ \\
Asymptomatic & \\
& \\
Radiographic findings $(N=22)$ & $9(41 \%)$ \\
Nodule & $9(41 \%)$ \\
Nodules & $3(14 \%)$ \\
Consolidation & $1(4 \%)$ \\
Consolidations & \\
Predisposing factors $(N=21)$ & $10(43 \%)$ \\
History of prior surgery & $2(8 \%)$ \\
Deep venous thrombosis & $2(8 \%)$ \\
CHF & \\
Sites of biopsy (N=22) & $13(60 \%)$ \\
RLL & $1(4 \%)$ \\
RUL & $2(10 \%)$ \\
RML & $3(13 \%)$ \\
LLL & $3(13 \%)$ \\
LUL &
\end{tabular}

RUL, right upper lobe; RML, right middle lobe; RLL, right lower lobe; LUL, left upper lobe; LLL, left lower lobe; M, male; F, female; SOB, shortness of breath.

There were 12 male and 11 female patients, ranging in age from 35 to 78 years (mean age 54.2 years; median age 48 years). Cough and shortness of breath were the most common clinical presentations, with a significant number being asymptomatic with their lesions identified on screening chest radiographs and/or CT scans. Hemoptysis and hemosputum were reported in only four cases (14\%). 'Pneumonia' was a presenting symptom in three cases.

The radiographic findings were available in 22 patients, all of whom had CT scans. In $82 \%$ of cases the lesion under investigation was regarded as a nodule/mass or nodules/masses (nine cases each). Bilateral lung nodules were specifically described in five instances. In three cases (14\%) radiographic studies indicated a localized alveolar consolidation, whereas in one case bilateral consolidations were described. The radiologist's impression was reported in 22 cases, with 17 cases $(77 \%)$ indicating a primary need to exclude neoplasia. In three cases $(14 \%)$ a concern for pneumonia or granulomatous disease was indicated. In only two instances (9\%) was pulmonary infarct considered in the differential diagnosis.

Predisposing factors for pulmonary thromboembolic disease were investigated in 21 cases. In only two cases was there a history of deep venous thrombosis manifested by lower extremity disease. Ten patients however gave a history of recent surgery (within the year) whereas two patients
Table 2 Pathologic findings in 23 thromboembolic pulmonary infarcts

\begin{tabular}{lr} 
Low magnification configuration of infarct & \\
Wedge/triangular & $6(26 \%)$ \\
Geographic & $8(35 \%)$ \\
Round & $4(17 \%)$ \\
Mixed & $5(22 \%)$ \\
Granulomatoid rim & $17(74 \%)$ \\
Airspace giant cells & $4(17 \%)$ \\
Basophilic necrosis & $12(52 \%)$ \\
Thromboemboli & \\
Without vascular inflammation & $11(47 \%)$ \\
With vascular inflammation & $12(52 \%)$ \\
Marked vascular inflammation & $5(22 \%)$ \\
Squamous metaplasia & $8(35 \%)$ \\
\hline
\end{tabular}

reported a history of congestive heart failure. One patient each gave a history of intravenous drug abuse, chemoradiation for small-cell carcinoma, and protein $\mathrm{C}$ deficiency. Patients had surgery for a variety of conditions, most often primary extrathoracic carcinomas $(N=3)$; others included thoracic bullectomies $(N=2)$ and abdominal surgery for obstruction due to volvulus and Crohn's disease (one each). Subsequent studies at the referring institutions after lung biopsy revealed right atrial mural thrombi in four cases, evidence of lower extremity venous thrombi in eight cases, and pulmonary angiograms and/or repeat HRCT scans compatible with pulmonary thromboemboli in four cases. In summary, with $2 / 23$ cases having a known history of deep venous thrombosis and 16/23 cases having clinical or radiographic data supporting a diagnosis of infarct after lung biopsy, 18/23 (78\%) of our patient group had clinical information that helped support the histologic diagnosis of a pulmonary infarct.

The referring pathologists provided a diagnosis in 16 of the 23 cases. In seven cases (44\%), the original diagnosis was necrotizing granulomatous disease, probably due to infection. In three cases (19\%), an original diagnosis of Wegener's granulomatosis was entertained, whereas in two $(12 \%)$ instances, the referring pathologists were concerned about an infiltrating malignancy associated with extensive necrosis. In only four cases (25\%) did the referring pathologist consider the possibility of a pulmonary infarct.

\section{Pathologic Findings}

The pathologic features of these 23 thromboembolic pulmonary infarcts are summarized in Table 2. At low magnification, only six cases (26\%) demonstrated a prototypic wedge- or triangular-shaped pleural-based zone of infarction with overlying chronic fibrous pleuritis. In fact, the majority of cases (eight cases; 35\%) showed geographic regions of coagulative necrosis in the subpleural region 


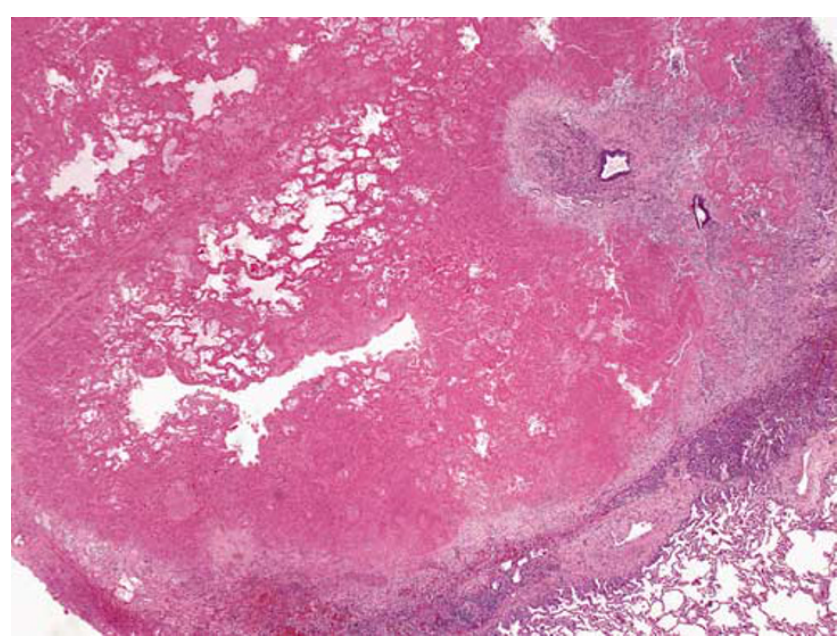

Figure 1 Pulmonary infarct with geographic necrosis. At left in this low-magnification photomicrograph, one can see the extensive coagulative necrosis that abuts the visceral pleura. At right one can identify a geographic pattern of necrosis with residual islands of pulmonary parenchyma, particularly around the bronchovascular bundle enveloped by the necrotic debris.

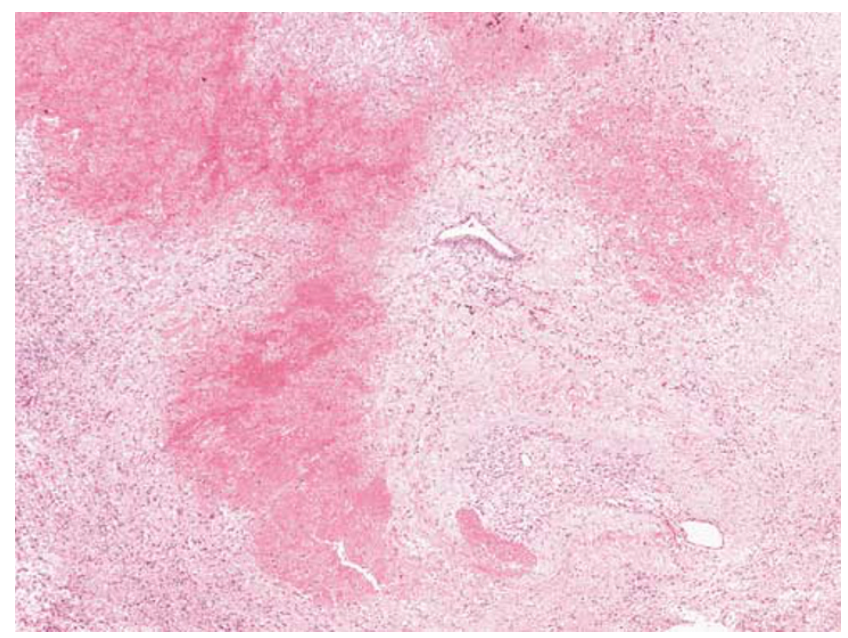

Figure 2 Pulmonary infarct with geographic necrosis. In many instances the geographic necrosis assumed an antler-like configuration with eosinophilic hemorrhagic coagulative necrosis crisscrossing the pulmonary parenchyma associated with a histiocytic reaction at its margin and squamous metaplasia of small bronchioles.

having a serpentine distribution, with islands of viable lung parenchyma often surrounded by broad swaths of eosinophilic necrosis (Figures 1 and 2). In four cases $(17 \%)$, the low power configuration of the pulmonary infarct was round, with these cases having a thick fibrous capsule enveloping a nearspherical region of necrosis (Figure 3). In five cases $(22 \%)$ the pattern was mixed, with four cases demonstrating segments with both a rounded and geographic configuration, whereas in one case the infarct had a triangular and geographic configuration. In all instances, the area of infarction showed coagulative necrosis of pulmonary parenchyma

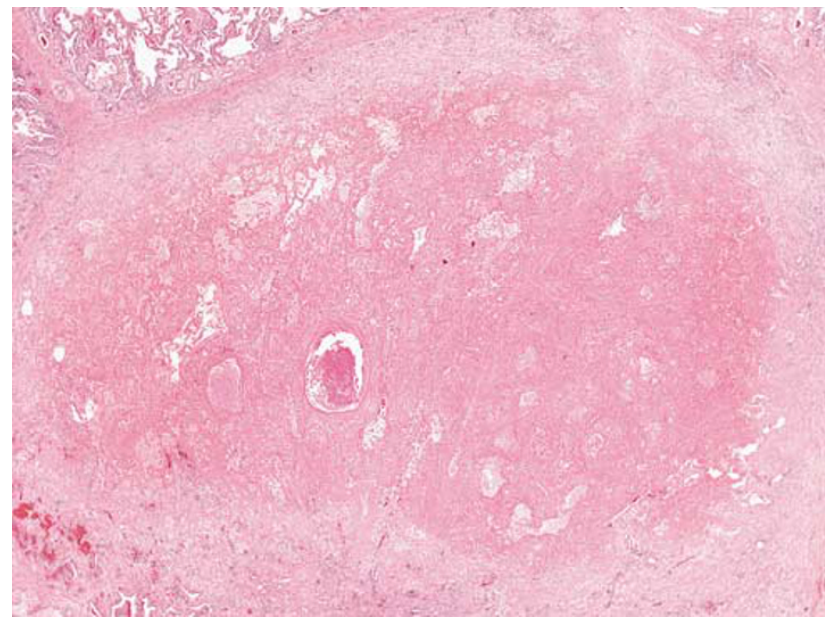

Figure 3 Pulmonary infarct with round configuration. This pulmonary infarct assumed a more oblong appearance, with central hemorrhagic and coagulative necrosis associated with a peripheral rim of dense fibrous connective tissue.

associated with extensive areas of hemorrhage. In these zones, the intact alveolar skeleton could be discerned despite the cellular components being largely necrotic. In 16 cases, the regions of necrosis crossed interlobular septa into adjacent parenchyma. At the edge of these regions of necrosis, there was a prominent reparative reaction with granulation tissue filling airspaces and a thickened interstitium. In 17 cases (74\%), there was a well-defined histiocytic and foamy macrophage margin at the edge of the coagulative necrosis that had a palisaded configuration (Figure 4). This palisaded reaction was largely composed of histiocytes, foamy macrophages, hemosiderin-laden macrophages, and activated fibroblasts/myofibroblasts. Giant cells were not present, although in four instances $(17 \%)$ giant cells were present in adjacent airspaces, containing cholesterol clefts and hemosiderin. These findings imparted a pseudogranulomatous quality to the zone of necrosis that was no doubt responsible for the referring diagnosis of granulomatous disease. Outside of the histiocytic reaction was an inflammatory margin that in most instances took the form of an organizing pneumonia with intra-airspace and interstitial myxoid plugs of young granulation tissue associated with the proliferation of fibroblasts and myofibroblasts, and a lymphoplasmacytic infiltrate. Hemosiderin-laden macrophages, hematoidin pigment, and often-fresh hemorrhage were admixed and in two cases dystrophic calcification was seen both at the margin and within the region of infarct. In six cases $(26 \%)$, the organizing rim coexisted with a thickened fibrous capsule that enveloped the region of hemorrhagic coagulative necrosis and in three cases $(13 \%)$ a palisaded histiocytic reaction and organizing pneumonia was not seen at all. Instead, the region of coagulative necrosis was encircled by a thick bland fibrous capsule associated with a chronic inflammatory infiltrate of lymphocytes, plasma cells, 

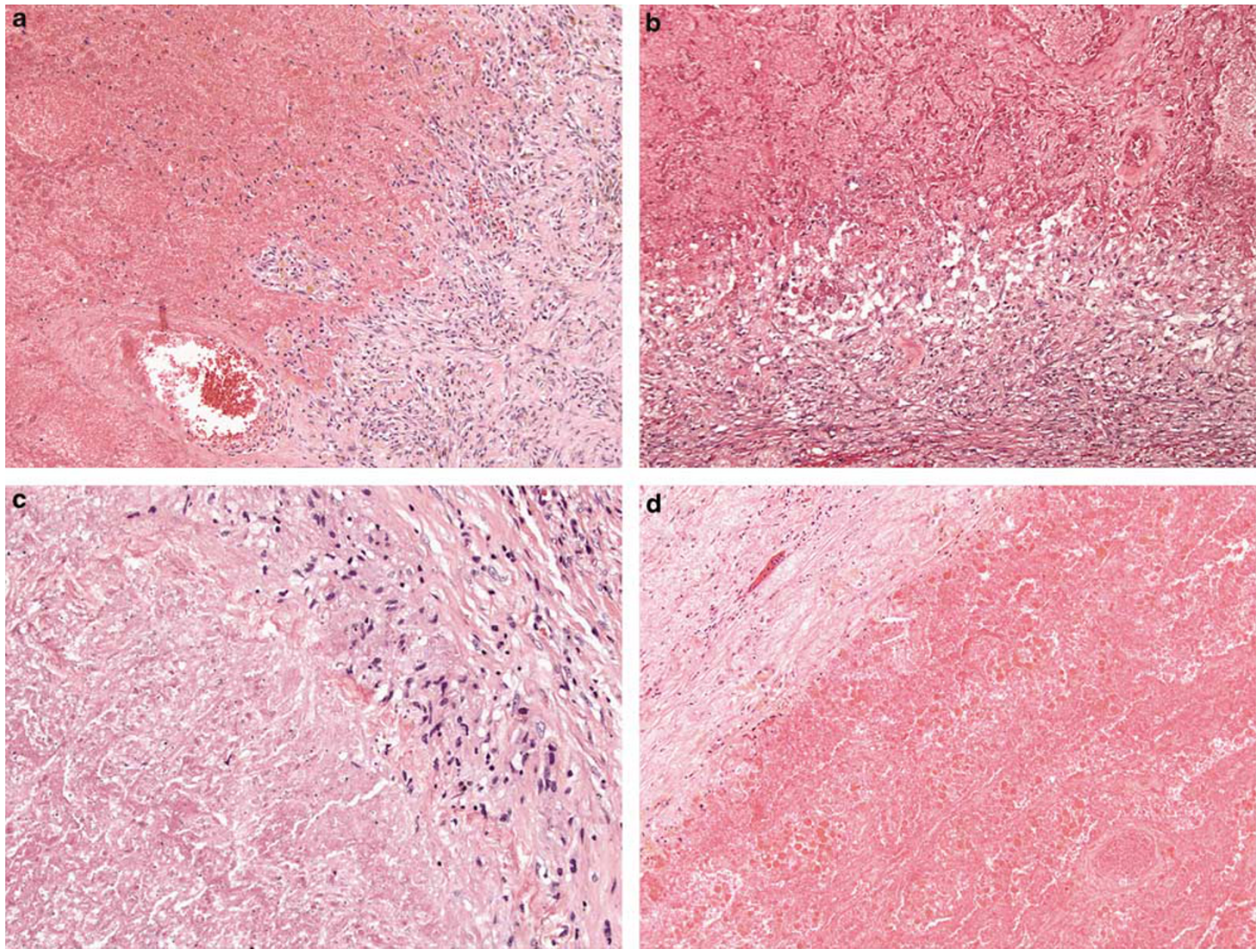

Figure 4 Margins of pulmonary infarcts. In most instances, the margins of the necrosis were lined by granulation tissue associated with a chronic inflammatory infiltrate. In many instances, the fibroblasts and myofibroblasts of the granulation tissue assumed a perpendicular orientation to the necrosis resulting in a pseudogranulomatous appearance (a). In other instances, the granulation tissue contained abundant foamy macrophages that combined with the mononuclear infiltrate resulting in a pseudogranulomatoid configuration (b). At lower left (c), the necrosis has prominent epithelioid histiocytes at its margin, a feature most often seen in older infarcts, although in panel $\mathbf{d}$, the infarcted lung was juxtaposed to a dense fibrous capsule without a significant inflammatory reaction.

and histiocytes. In eight instances, squamous metaplasia was observed (Figure 5) with reactive alveolar pneumocyte hyperplasia present in two cases. These cases were the basis for the referring pathologist's concern for malignancy.

In the zones of coagulative necrosis, 12 cases (52\%) had areas of basophilic staining at low magnification (Figure 6). At higher magnification, these zones of necrosis were characterized by granular basophilic karyorrhectic debris (leukocytoclasis) that often extended to the margins of the pulmonary infarct and were associated with a patchy neutrophilic infiltrate. These 'blue' zones represented karyorrhectic debris originating from neutrophils and necrotic parenchymal cells. Such zones of necrobiosis combined with the presence of giant cells and changes in the blood vessels (see below) were the basis for histologic concern for Wegener's granulomatosis (Figure 7).

Pulmonary thromboemboli were found in all 23 cases within muscular pulmonary arteries. In only five instances were thrombi also present in pulmonary veins. What was most striking about the pulmonary thromboemboli was mural inflammation in 13 instances (56\%) (Figure 8). This reflected the presence of lymphocytes, plasma cells, and rare neutrophils in the subendothelial region and media of these pulmonary arteries. In five instances, the infiltrate was prominent, splaying apart the smooth muscle of arterioles without necrosis of the vessel wall or fragmentation of arterial elastica. Nonetheless, this intense transmural inflammatory infiltrate in 5 of the 23 cases raised the possibility of vasculitis as the cause for the pulmonary infarct and necrosis. Parenthetically, antineutrophil antibodies were absent in four cases for which data were available.

In eight instances, respiratory bronchiolitis with emphysematous change was seen in the adjacent lung parenchyma whereas in one case follicular bronchiectasis was also observed. 


\section{Discussion}

Descriptions of pulmonary infarcts in their acute stages are standard elements of medical student lectures on hemostasis and coagulation. ${ }^{2-8}$ These wedge-shaped subpleural zones of hemorrhage and coagulative necrosis are rare due to the triple supply of oxygen to the lung from the bronchial and pulmonary arteries and air of the tracheobronchial tree as well as the intrinsically high fibrinolytic activity of pulmonary endothelial cells. ${ }^{9-11}$ Nonetheless, in the setting of chronic cardiovascular or pulmonary disease, thromboembolic infarcts occur and are common in autopsy series, often representing a major cause of sudden death..$^{7,8,12}$

The genesis of this study originated in the escalating number of surgically resected nodules sent for consultation with a wide differential diagnosis often not including thromboembolic pulmonary infarcts. We reviewed 23 cases of resected pulmonary infarcts sent for consultation, 10 within the last 2 years, to identify the morphologic findings that lead to this confusion. Several observations could be made. First the classical teaching that infarcts were triangular or wedge shaped proved not to be true in this series, possibly due to their organized nature, although sampling bias for biopsy of atypical lesions needs to be considered. ${ }^{1-3,8,11}$ Although these lesions were pleural based and associated with overlying pleuritis, the majority of these nodules were round with a thick fibrous capsule or had a geographic pattern of necrosis that crisscrossed the parenchyma and traversed interlobular septa. The irregularity in the pattern of necrosis could potentially be

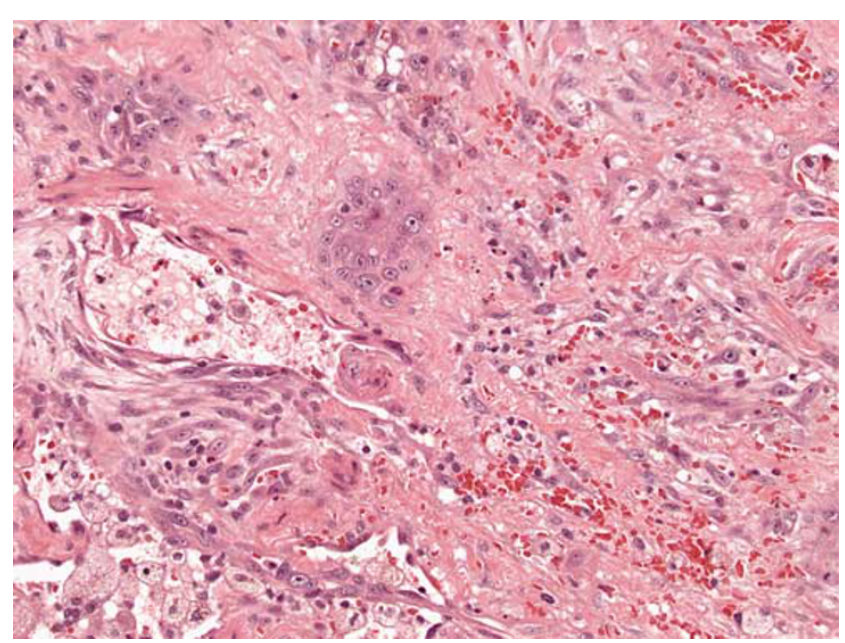

Figure 5 Pulmonary infarct with squamous metaplasia. Squamous metaplasia was a common finding and often showed significant reactive changes that can lead to a differential diagnosis of malignancy.

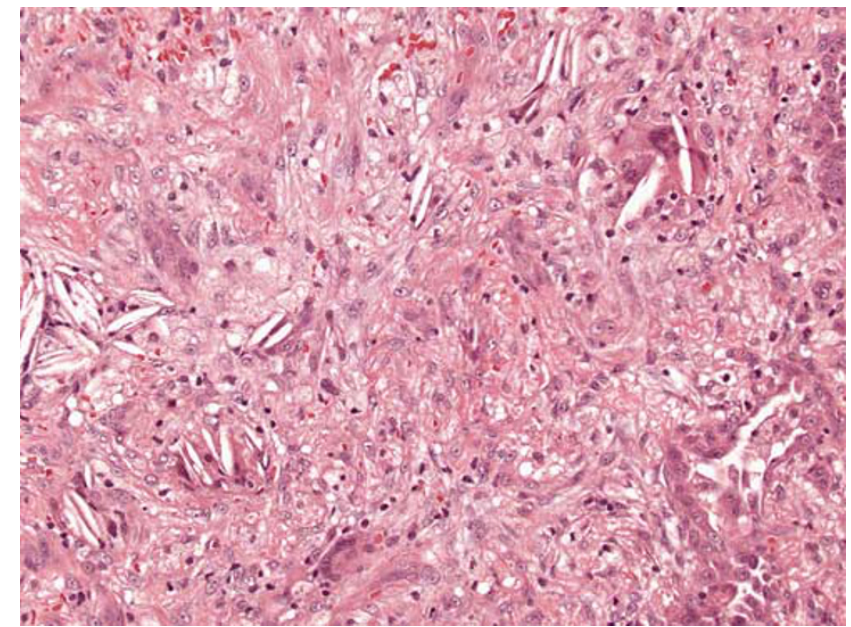

Figure 7 Pulmonary infarct with giant cell reaction. Outside of the rim of repair, giant cells, many with prominent cholesterol clefts were present. This probably represented a nonspecific reaction to degenerating red blood cells and cell debris.
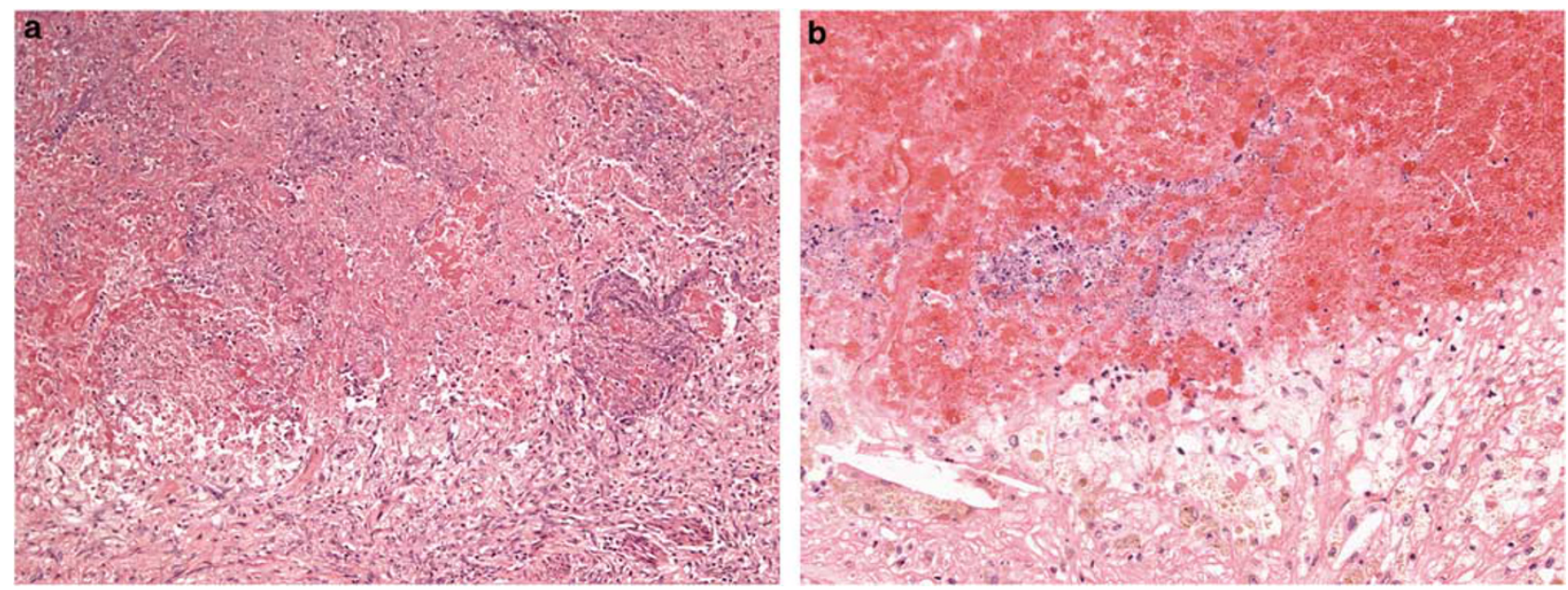

Figure 6 Pulmonary infarct with basophilic necrosis. At left, the margin of the infarct is associated with prominent foamy and hemosiderin-laden macrophages with a reparative reaction. In this instance, the necrosis has a distinctive basophilic appearance that at higher power (at right) has the granular karyorrhectic appearance of leukocytoclastic necrosis, here with a rim of foamy macrophages. 
684

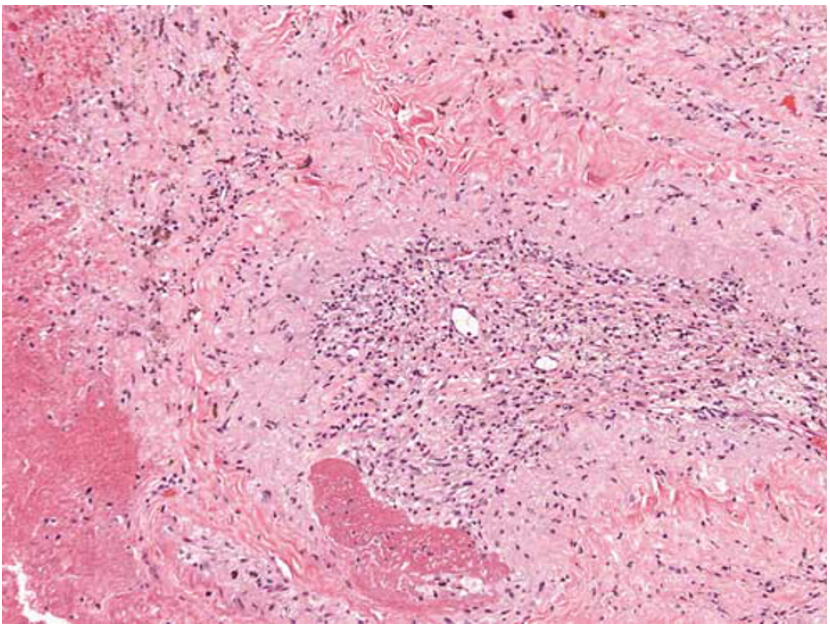

Figure 8 Pulmonary infarct with vascular inflammation. In over half the cases, inflammation of thrombosed and recanalized blood vessels was seen, which in rare instances was accompanied by necrosis of the vessel wall, particularly when these vessels were juxtaposed to zones of necrosis. Such changes often resulted in a differential diagnosis of vasculitis.

explained by showers of thromboemboli into the affected lobules, poor perfusion of chronically altered parenchyma due to emphysema, anatomic variants, or coexistent venous obstruction secondary to complicating diseases such as congestive heart failure or chronic obstructive lung disease. In contrast to the pathology literature, it is well recognized in thoracic radiology that infarcts may have an irregular and spiculated appearance in a minority of instances. ${ }^{4-7,9,12-16}$ In $52 \%$ of cases, the coagulative necrosis of parenchyma was punctuated with basophilic necrosis where parenchymal cells, histiocytes, and leukocytes were undergoing degeneration resulting in low power streaks of 'leukocytoclasis' or 'pathergic necrosis' much as one sees in Wegener's granulomatosis. ${ }^{17}$ This hematoxyphilic change to the zones of coagulative necrosis and hemorrhage was not predicted given the usual emphasis on hemorrhage and bland ischemic necrosis of parenchyma in infarcts.

What also lead to a diagnosis of necrotizing granuloma in the vast majority of cases was the presence of a pseudopalisade of histiocytes, foamy and hemosiderin-laden macrophages, organizing granulation tissues, and occasional giant cells abutting the zones of coagulative necrosis. The histiocytes lacked epithelioid transformation and giant cells were not present at the edge of the necrosis although they were present in adjacent airspaces as cholesterol-laden multinucleated macrophages. Although infarct-like necrosis can be seen in necrotizing granulomas, the distinct granulomatous reaction of activated epithelioid histiocytes and frequent marginal giant cells can be discriminatory. ${ }^{18}$ For these reasons, major referring diagnoses were infectious granuloma followed by vasculitis, primarily Wegener's granulomatosis.
The consideration for Wegener's granulomatosis points to another aspect of pulmonary infarcts and their occlusive thromboemboli. As part of the organization of pulmonary thromboemboli, there is an influx of mononuclear cells into the vessel walls that can be sufficiently intense to mimic a vasculitis especially when seen in the context of mural or subendothelial fibrin deposits resembling fibrinoid necrosis. When combined with a geographic pattern of necrosis, a pseudogranulomatous histiocytic rim, and vascular inflammation, Wegener's disease or infection is a logical consideration. The appearance of these inflamed pulmonary arteries has not been emphasized in discussions of infarcts, except to note that such thromboemboli may be 'septic'—caused by microbial emboli..$^{1,7,8}$ The observation that some infarcts may cavitate can cause further confusion with Wegener's disease. ${ }^{19-22}$

As part of the organization of pulmonary infarcts, the adjacent alveolar pneumocytes may become quite hyperplastic and atypical. Similarly the epithelium of bronchi and bronchioles in the region of infarct may show exuberant squamous metaplasia to the point where histologic consideration has been given to squamous carcinoma. Such epithelial atypia is a well-reported problem in fine needle aspirations and other cytologic preparations with pulmonary infarcts as such cases have been overcalled as malignant. ${ }^{4,10,21}$ This was a problem in several of our consultation cases too.

The vast number of pulmonary infarcts never come to biopsy because of their clinical presentation or because the radiology of pulmonary thromboemboli and infarcts are so well described., ${ }^{4,7,13}$ Nonetheless, in only three cases where CT scan reports were available did the radiologist raise the possibility of thromboemboli and infarction, possibly reflecting the peculiar low-magnification characteristics that we have described. Radiologic descriptions highlight the pleural-based nature of the lesion, often seen in the lower lobes, with the apex of the wedge-shaped infarct pointing toward the thrombosed vessel and a convex border (Hampton's hump) reflecting the hemorrhage and bulging of the interlobular septa and/or compressed lung parenchyma. ${ }^{4,5,9,11,13,15}$ Coinciding with the atypical radiologic presentation, patients did not characteristically present with hemoptysis, chest pain, and pleurisy with the vast majority of patients identified by nonspecific complaints of cough or shortness of breath or by screening radiographic exams for metastatic carcinoma. A significant percentage of our patients did, however, have risk factors for pulmonary thromboemboli and infarcts, confirmed in postoperative studies.

Our study focused exclusively on thromboembolic pulmonary infarcts. We did not include other known causes of vascular occlusion and parenchymal necrosis, including infection such as angioinvasive mycoses, angioinfiltrative primary or secondary malignancies, vasculitides, amyloidosis, 
lobar torsion, air or amniotic fluid emboli, or infarcts due to foreign bodies including iatrogenic ones such as vascular catheters. ${ }^{7}$ These possibilities, among others, would need to be included in the differential diagnosis of pulmonary infarcts.

In summary, pulmonary infarcts are rarely seen in the surgical pathology suite being much more common on the autopsy service. Although pleural based and hemorrhagic, they may have features that differ from classical descriptions including a predominant round configuration or geographic pattern of necrosis, foci of palisaded histiocytes at their margin, vascular inflammation, adjacent reactive alveolar pneumocyte, and squamous metaplasia with atypia, all of which may cause diagnostic confusion with infectious granulomas, vasculitis including Wegener's granulomatosis and neoplasia.

\section{Acknowledgement}

I thank Diana Winters for secretarial assistance and Linda Shab and Tom Bauer for photographic aid and, special thanks to CBC who fooled me with this diagnosis, more than once.

\section{References}

1 Cotran R. Robbins and Cotran Pathologic Basis of Disease 7th edn. Elsevier Saunders: Philadelphia, PA, 1999;742-743, 1364-5pp.

2 Castleman B. Healed pulmonary infarcts. Arch Pathol 1940;30:130-142.

3 Dunnill MS. The pathology of pulmonary embolism. Br J Surg 1968;55:790-794.

4 George CJ, Tazelaar HD, Swensen SJ, et al. Clinicoradiological features of pulmonary infarctions mimicking lung cancer. Mayo Clin Proc 2004;79:895-898.

5 Hampton AO, Castleman B. Correlation of postmortem chest teleroent-genograms with autospy findings. Am J Roentgenol Radium Ther 1940;43:305-326.

6 Morpurgo M, Schmid C. The spectrum of pulmonary embolism. Clinicopathologic correlations. Chest 1995;107:18S-20S.

7 Parambil JG, Savci CD, Tazelaar HD, et al. Causes and presenting features of pulmonary infarctions in 43 cases identified by surgical lung biopsy. Chest 2005; 127:1178-1183.

8 Wagenvoort CA. Pathology of pulmonary thromboembolism. Chest 1995;107:10S-17S.

9 Ren H, Kuhlman JE, Hruban RH, et al. CT of inflationfixed lungs: wedge-shaped density and vascular sign in the diagnosis of infarction. J Comput Assist Tomogr 1990;14:82-86.

10 Schraufnagel DE, Tsao MS, Yao YT, et al. Factors associated with pulmonary infarction. A discriminant analysis study. Am J Clin Pathol 1985;84:15-18.

11 Tsao MS, Schraufnagel D, Wang NS. Pathogenesis of pulmonary infarction. Am J Med 1982;72:599-606.

12 Johnson PT, Wechsler RJ, Salazar AM, et al. Spiral CT of acute pulmonary thromboembolism: evaluation of pleuroparenchymal abnormalities. J Comput Assist Tomogr 1999;23:369-373.

13 Balakrishnan J, Meziane MA, Siegelman SS, et al. Pulmonary infarction: CT appearance with pathologic correlation. J Comput Assist Tomogr 1989;13:941-945.

14 Katsumura Y, Ohtsubo KI. Correlation between clinical and pathological features of pulmonary thromboemboli and the development of infarcts. Respirology 1998;3:203-206.

15 Lourie GL, Pizzo SV, Ravin C, et al. Experimental pulmonary infarction in dogs: a comparison of chest radiography and computed tomography. Invest Radiol 1982;17:224-232.

16 Mathis G, Dirschmid K. Pulmonary infarction: sonographic appearance with pathologic correlation. Eur J Radiol 1993;17:170-174.

17 Bloodworth J, Tomashefski Jr JF. Localised pulmonary metastatic calcification associated with pulmonary artery obstruction. Thorax 1992;47:174-178.

18 Ulbright TM, Katzenstein AL. Solitary necrotizing granulomas of the lung: differentiating features and etiology. Am J Surg Pathol 1980;4:13-28.

19 Libby LS, King TE, LaForce FM, et al. Pulmonary cavitation following pulmonary infarction. Medicine (Baltimore) 1985;64:342-348.

20 Morgenthaler TI, Ryu JH, Utz JP. Cavitary pulmonary infarct in immunocompromised hosts. Mayo Clin Proc 1995;70:66-68.

21 Redline S, Tomashefski Jr JF, Altose MD. Cavitating lung infarction after bland pulmonary thromboembolism in patients with the adult respiratory distress syndrome. Thorax 1985;40:915-919.

22 Wick MR, Ritter JH, Schuller D. Ruptured pulmonary infarction: a rare, fatal complication of thromboembolic disease. Mayo Clin Proc 2000;75:639-642. 